\title{
Modification of $\Delta \log R$ method and Nonlinear Regression Application for Total Organic Carbon Content Estimation from Well Logs
}

\author{
Can Polat ${ }^{1}$ (D) Tuna Eren ${ }^{2}$ \\ ${ }^{1}$ Izmir Katip Celebi University, Department of Petroleum and Natural Gas Engineering, Izmir, Turkey \\ ${ }^{2}$ Eni International Resources Limited, Ankara, Turkey
}

\section{ABST RACT}

$\triangle$ $\log \mathrm{R}$ method is one of the most widely utilized techniques for estimation of Total Organic Carbon (TOC) content form well logs. The traditional $\Delta \log R$ method reveals the assumption of linear relationship between logarithmic resistivity and porosity log. In this study, the method is modified by means of integral calculus to acquire the actual trend between logarithmic resistivity and porosity log. Furthermore, unlike to the traditional method, the maturity is additionally represented with logarithm of organic maturation temperature. Nonlinear regression is applied to optimize the unclear organized parameters required for computation of TOC content. The final forms of the equations are observed to be appropriate for the nonlinear regression application. The TOC estimations are observed to be improved with the modified versions in case traditional methods are unsatisfactory due to the related assumption expressed above. With the introduced methodology the TOC of unconventional reservoirs and source rocks can be more accurately calculated.

\author{
Article History: \\ Received: 2021/03/08 \\ Accepted: 2021/06/22 \\ Online: 2021/06/30 \\ Correspondence to: CanPolat, \\ Izmir Katip CelebiUniversity, \\ DepartmentofPetroleumand \\ Natural Gas Engineering, Izmir, \\ Turkey \\ E-mail:can.polat@ikcu.edu.tr; \\ Phone: +905057376766 .
}

Keywords: Total organic carbon, Well $\operatorname{logs}, \Delta \log R$ method, Nonlinear regression, Unconventional resources

\section{INTRODUCTION}

$\mathrm{R}$ ecent studies reveal that unconventional resources are gaining importance with the developments in the utilization of these resources. The unconventional reservoirs are handled in terms of their hydrocarbons in place with the calculation methodologies valid for petroleum systems [1]. These formations are mostly tiny grained, rich in total organic content and are sedimentary rocks [2]. The formations of unconventional reservoirs are usually made up of shales and alike rocks. Total Organic Carbon (TOC) is a term that is a property used to determine whether such formations can be economically viable or not [3]. For instance, the gas content in formations is found to have a linear relationship with the TOC [4]. Generally speaking, Total Organic Carbon (TOC) content value that is higher than $2.0 \mathrm{wt} \%$ is an important aspect in turning shale gas resources into a reservoir [5]. Ahmed and Meehan in their study stated that production performance of unconventional wells is highly dependent on the accurate placement of the horizontal wells and application of the correct fracture stages in the reservoir intervals with high rock quality properties and prolific production capability
[6]. However, the significance of reservoir's fracture capability and as important as this the well placement into the highest total organic content zone is not comprehended as required. Therefore, the TOC estimation is a very important aspect to be taken into consideration for successful unconventional campaign reinstatements.

In source rocks, organic carbon is present in kerogen, bitumen and hydrocarbons [7]. Kerogen maturation brings the generation of oil and gas in the source rock. TOC determination is essential for proper evaluation of both unconventional reservoirs and source rocks. The possibility of generation of sufficient quantity of oil and gas increases with TOC content. According to Thomas, the weight percentage of the organic carbon in the source rock should be at least $0.5 \mathrm{wt} \%$ to regard the rock as a fair source rock from the point of potentiality in generating hydrocarbons [8]. Besides, with kerogen maturation the carbon concentration rises [1].

According to Steiner et al., TOC from a core sample can be determined mainly in two ways [7]. In the first 
procedure (LECO-based measurement), the inorganic carbon is removed by acidification, and the remaining organic carbon is detected by carbon analyzer by means of combusting the organic carbon to $\mathrm{CO} 2$. In the second procedure (Rock-Eval method), a pulverized rock sample is subject to step-wise pyrolysis temperature rise. Perpetual monitoring of $\mathrm{CO} 2$ and $\mathrm{CO}$ during pyrolysis and oxidation make it possible to differentiate between organic and inorganic carbon oxides. The loss of some hydrocarbons in consequence of drying and washing process is the disadvantageous point of the first method. Reliance on the assumption that the weight percent of carbon in hydrocarbon is 0.83 and inability to discern the origin of $\mathrm{CO}$ and $\mathrm{CO} 2$ in case of overlapping related to pyrolysis and oxidation are the negative sides of the second procedure. It is possible to acquire accurate TOC estimations by means of these measurement techniques. However, the stated TOC estimations are time consuming and costly [9].

One of the most common methods to determine TOC is the use of logging data that belongs to the reservoir interval [10]. The advantage point in using well logs is that they provide continuance in TOC content estimation. Resistivity logs being indicators for hydrocarbons in the pore spaces can be evaluated with porosity logs that can be utilized to reveal the kerogen existence. One of the early research works in relation to that is the study conducted by Passey et al. [11]. In their study they proposed a practical method known as $\triangle \log \mathrm{R}$ method, in which they used the well log data to identify the TOC in organic rich rocks. The method involves the process of overlapping the resistivity log displayed on a logarithmic scale with one of the porosity logs to reveal the baseline corresponding to nil carbon content. TOC is estimated based on the departures from this baseline. Besides, uranium content deducted from natural gamma spectroscopy can be indicative for TOC. Uranium content is evaluated with thorium or potassium content to develop correlations for TOC estimation [10]. It is also proved that the density log can be properly correlated with TOC [10]. Furthermore, in their study Decker et al. stated that rig site gas content measurements are proven to be more effective through density log measurements to quantify the gas content of the shale intervals [12].

The $\Delta \operatorname{LogR}$ method is a practical and commonly utilized method to estimate TOC content. Based on the range of the training data, the traditional approach in $\Delta$ LogR method may yield considerably improper estimates of TOC for some intervals. This study involves the modification of the traditional formulation. The modified method is utilized with the intend of improving the estimation where traditional method fails due to the reason mentioned above.

Unconventional resource exploration is gaining utmost importance globally. TOC is a terminology which is the very starting point for the determination of whether such resources are economically viable or not. For this reason, one of the most significant contributions of present study is to reveal the TOC content of the unconventional resources utilizing the methodologies by means of making use of the well logs is introduced in the scope of this research work. The present research study is also important for its capability to test the source rocks' potentiality to generate hydrocarbons.

\section{METHODS}

\section{Traditional Formulation}

The resistivity and porosity curves in the log interval corresponding to the zone free of organic matter can be coincided by scaling the porosity curve. The line passing through the coincided curves located in the interval corresponding to the zone free of organic matter is known as baseline. The magnitude of departure of resistivity and porosity curves from the baseline and the determined level of organic maturity provides the total organic carbon [11] (Eq. (1) and Eq. (2)).

$$
T O C=\Delta \log R \times 10^{(a+b \times L O M)}
$$

$\Delta \log R$ can be expressed as the log of the difference between the resistivity reading from the log and the resistivity value obtained by means of the curve passing through baseline resistivity and transit time values with a slope of p (Eq. (2)). Here, difference in transit time is multiplied by a factor $\mathrm{p}$ to reveal the equivalent difference in terms of logarithm of resistivity. In this method, linear relationship between logarithmic resistivity and porosity log is assumed. Actually, the relationship is not strongly linear. The validity of the assumption depends on the range of the porosity log. The departures from the linearity may lead to requirement of checking the validity of this assumption.

$$
\Delta \log R=\log _{10}\left(R / R_{l}\right)+p \times\left(\Delta t-\Delta t_{l}\right)
$$

The extended form of Eq. (1) is as follows

$$
T O C=\Delta \log (R)+a_{1} \times\left(\Delta t-a_{2}\right) \times 10^{\left(a_{3}+a_{4} \times L O M\right)}
$$

where

$$
\begin{aligned}
& a_{1}=p \\
& a_{2}=\Delta t_{l}+\log _{10}\left(R_{l}\right) / p
\end{aligned}
$$

$R, \Delta t, R_{p} \Delta t_{p}$ and $L O M$ denote the resistivity, and transit time readings on the corresponding logs, resistivity and transit time reading on the baseline, and level of organic maturity, respectively.

In this study, the parameters $a_{1}, a_{2}, a_{3}$ and $a_{4}$ were optimized by means of application of the Levenberg-Marquardt algorithm [13] [14]. 


\section{Modified Version}

A more proper relationship can be obtained with the help of Archie equation [15] (Eq. (6)) which associates the resistivity of the brine saturated rock to the porosity.

$$
R_{o}=R_{w} / \phi^{m}
$$

The porosity can be expressed as a function of transit time. The resultant equation is:

$$
R_{o}=R_{w} /\left[\left(\Delta t-\Delta t_{m}\right) /\left(\Delta t_{f}-\Delta t_{m}\right)\right]^{m}
$$

$\Delta t_{m}, \Delta t_{\rho} R_{w}, R_{o}$ and $m$ represent transit time of matrix and fluid (brine) in the pores, resistivity of the brine, and the brine saturated zone and cementation exponent of the rock, respectively.

Eq. (7) can be utilized to calculate the resistivity for the sample free from organic matter at the considered transit time. Taking the logarithm of both sides in Eq. (7), and derivative with respect the transit time, gives the following equations (Eq. (8) and Eq. (9))

$$
\begin{aligned}
& \log _{10} R_{o}=\log _{10}\left\{R_{w} /\left[\left(\Delta t-\Delta t_{m}\right) /\left(\Delta t_{f}-\Delta t_{m}\right)\right]^{m}\right\} \\
& \frac{d \log _{10} R_{o}}{d \Delta t}=-\frac{1}{\ln 10} \frac{m}{\Delta t-\Delta t_{m}}
\end{aligned}
$$

Eq. (9) can be used to compute the slope of the curve at the considered transit time. In the study of Wang et al., $p$ in Eq. (2) is replaced with the expression on the right hand side of Eq. (9) to avoid the dependency on the assumption of linear relationship [16]. However, this approach may cause excessive magnitudes for the slope term and negative $\Delta \log R$ values accordingly. A proper relationship between logarithmic resistivity and transit time can be obtained by taking the integral of both sides in Eq. (9). The starting points of the integral are logarithmic resistivity and transit time readings corresponding to the baseline.

$$
\int_{\log _{10} R_{l}}^{\log _{10} R_{o}} d \log _{10} R_{o}=\int_{\Delta t_{l}}^{\Delta t}-\frac{1}{\ln 10} \frac{m}{\Delta t-\Delta t_{m}} \Delta t
$$

which gives

$$
\log _{10} R_{o}=\log _{10} R_{l}-\frac{m}{\ln 10} \ln \left(\frac{\Delta t-\Delta t_{m}}{\Delta t_{l}-\Delta t_{m}}\right)
$$

Ro in Eq. (10) can be considered as the resistivity of the sample free from organic matter. The magnitude of $\Delta \log R$ can be obtained subtracting logarithm of this value from the logarithm of resistivity value obtained from the well log.

$$
\Delta \log R=\log _{10} R-\log _{10} R_{o}
$$

Combination of Eq. (11) and Eq. (12) leads to the following equation

$$
\Delta \log R=\log _{10}\left(R / R_{l}\right)+\frac{m}{\ln 10} \ln \left(\frac{\Delta t-\Delta t_{m}}{\Delta t_{l}-\Delta t_{m}}\right)
$$

According to traditional formulation [11], TOC can be obtained using the Eq. (1) also given below

$$
T O C=\Delta \log R \times 10^{(a+b \times L O M)}
$$

Due to the lack of measurement of thermal maturity in the form of LOM, Wag et al. proposed using $\mathrm{T}_{\max }$ instead of LOM in Eq. (1) [16]. Then, equation for TOC has the following form

$$
T O C=\Delta \log R \times 10^{\left(a+b \times T_{\max }\right)}
$$

Note that the formulation presented by Wang et al. involves gamma ray values incorporated into within the $\Delta \log R$ equation and is expressed as follows.

$$
T O C=\left(a \Delta \log R+b\left(G R-G R_{l}\right)\right) \times 10^{\left(c+d \times T_{\max }\right)}
$$

Where $G R$ and $G R_{l}$ denote gamma ray and base line gamma ray readings, respectively. The unknown parameters $a, b, c$ and $d$ are to be optimized.

Utilization of Eq. (14) brought problems related to Jacobian matrix in the application nonlinear regression. The problem can be solved by using logarithm of $\mathrm{T}_{\max }$ instead of $\mathrm{T}_{\max }$ in Eq. (14). Utilization of Tmax instead of LOM requires somewhat strong linear relationship between $\mathrm{T}_{\max }$ and LOM. Indeed, it was observed that the strength of the linear relationship was preserved in logical intervals of $\mathrm{T}_{\max }$ (410-510 ${ }^{\circ} \mathrm{C}$ ) and LOM (0-20) in case of using logarithm of $\mathrm{T}_{\max }$ instead of $\mathrm{T}_{\max }$. Hence, the following equation is used to compute TOC in the absence of LOM values.

$$
T O C=\Delta \log R \times 10^{\left(a+b \times \log _{10}\left(T_{\max }\right)\right)}
$$

The simplified form is as follows

$$
T O C=\Delta \log R \times 10^{a} T_{\max }{ }^{b}
$$

The final form is as follows

$$
\text { TOC }=\left[\Delta \log (R)+\frac{m}{\ln 10} \ln \left(\Delta t-a_{1}\right)-a_{2}\right] \times 10^{a_{3}} T_{\max }^{a_{4}}
$$

Or

$$
\text { TOC }=\left[\Delta \log (R)+\frac{m}{\ln 10} \ln \left(\Delta t-a_{1}\right)-a_{2}\right] \times 10^{\left(a_{3}+a_{4} \times \text { LOM }\right)}
$$

Where

$$
\begin{aligned}
& a_{1}=\Delta t_{m} \\
& a_{2}=\frac{m}{\ln 10} \ln \left(\Delta t_{l}-\Delta t_{m}\right)+\log _{10} R_{l}
\end{aligned}
$$

In this study, the parameters $a_{1}, a_{2}, a_{3}$ and $a_{4}$ were optimized by means of application of the Levenberg-Marquardt algorithm. 
A specific interval can be considered to specify transit time and resistivity corresponding to the baseline and matrix transit time. The magnitude of these parameters can vary considerably based on the alteration in mineral composition. Hence, it is a more favorable approach to group them into one parameter for optimization rather than evaluating them explicitly. Different from those, the cementation exponent $(\mathrm{m})$ is not tried to be optimized as optimization process yields unrealistic m values. Rather, this parameter is evaluated explicitly. In this context, different logical cementation exponent values were tried and corresponding results were compared.

Combination of $\Delta \log R$ with other methods might be considered for improving estimation of TOC similar to the procedure presented in the study of Wang et al. [16] It should be checked whether the developed formulation is appropriate for nonlinear regression application. It was observed that the formulations utilized in this study were inappropriate for nonlinear regression application in case it involved additional unknown parameters. This study basically focuses on the improvement of the traditional $\Delta \log R$ method.

Density and neutron readings can be alternative to sonic readings for TOC estimation. In fact, sonic readings are more preferable since these readings are less adversely affected from improper hole conditions [11]. Therefore, sonic readings were evaluated to estimate the TOC in this study.

\section{Levenberg-Marquardt algorithm for Nonlinear Regression}

The related tool of Matlab software was utilized for nonlinear regression application. As a default, the tool utilizes Levenberg-Marquardt (LM) algorithm, a standard method for non-linear least squares problems. The algorithm takes advantage of steepest descent method or Gauss-Newton method based on the value of the damping factor. In case the algorithm tends to behave more like steepest descent method in relation to the reduced damping factor, its converge speed is slow but improvement in minimization is more guaranteed. Improvement in minimization leads to increase in the damping factor and the similarity to Gauss-Newton method and converge speed is advanced as a result [17]. Note that the initial values of the LM parameters are calculated using the related equations involving one or more of estimated baseline transit time and resistivity, matrix transit time, cementation factor, the slope, and initial values are kept constant.

In the regression process, the target is to minimize the following summation

$$
\sum_{i=1}^{m}\left[y_{i}-f\left(x_{i}, \beta\right)\right]^{2}
$$

Where $y_{i^{\prime}} f\left(x_{i} \beta\right), \beta$ represent the targeted (measured) and calculated resultant parameters, and parameter set to be optimized, respectively.

One of the following equations is used to compute the parameter vector $\delta$ to be added to vector $\beta$ to update it at each iteration [18].

$$
\begin{aligned}
& \left(J^{T} J+\lambda I\right) \delta=J^{T}[y-f(\beta)] \\
& \left(J^{T} J+\lambda \operatorname{diag}\left(J^{T} J\right)\right) \delta=J^{T}[y-f(\beta)]
\end{aligned}
$$

$I$ in Eq. (23) is the identity matrix. In the study of Fletcher, $I$ was replaced with the diagonal matrix comprised of the diagonal elements of $J^{T} J$ to improve the converge [19]. $J$ is the Jacobian matrix and expressed as follows:

$$
J_{i}=\frac{\partial f\left(x_{i}, \beta\right)}{\partial \beta}
$$

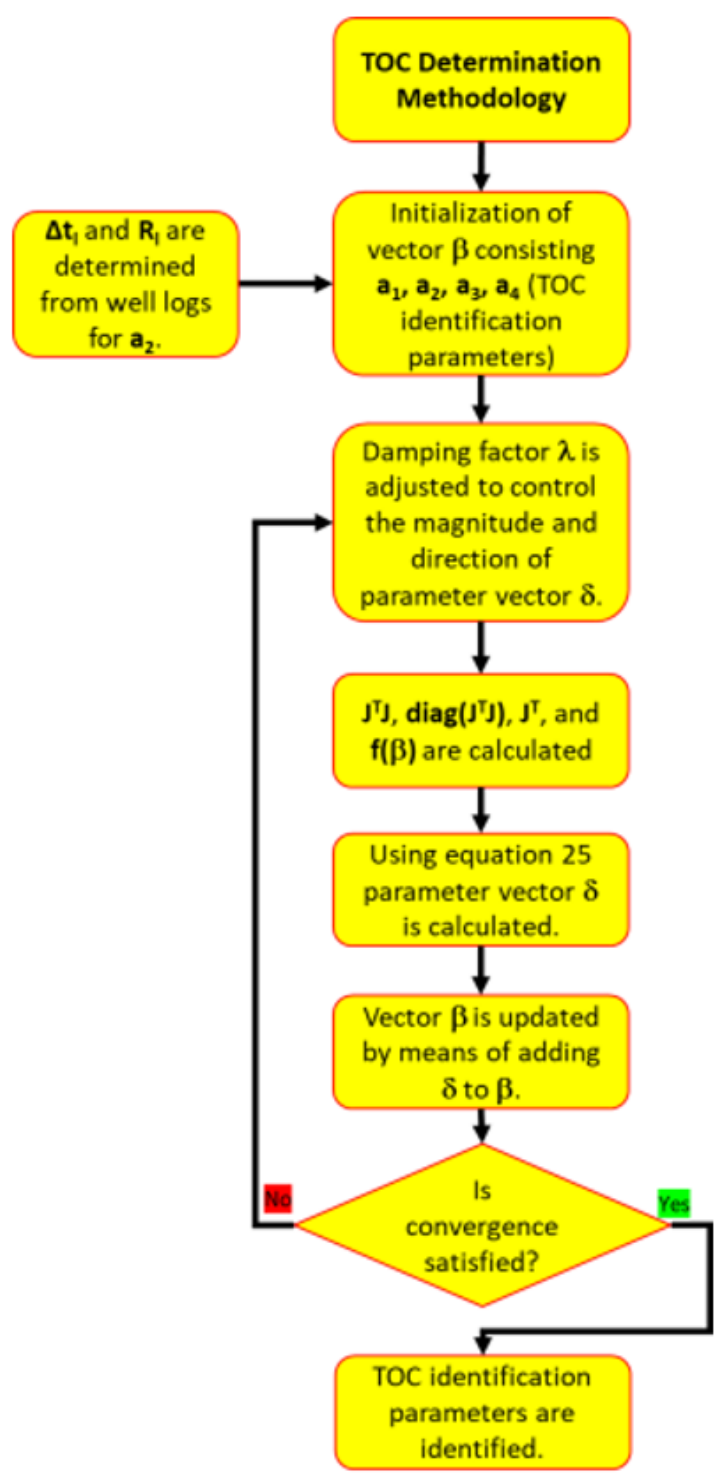

Figure 1. Methodology for Levenberg-Marquardt algorithm. 
Iterative calculation is required to optimize the parameters. At each iteration, Eq. (24) is computed for $\delta . J^{T} J$, the diagonal elements of $J^{T} J$ and $f(\beta)$ are computed using the latest vector $\beta$. The vector $\beta$ is updated at each iteration with the addition of new $\delta$. The damping factor $\lambda$ is adjusted at each iteration to control the direction and magnitude of $\delta$ (Fig. 1).

The algorithm detects a local minimum which might not be the global minimum. The initial value of the parameters to be optimized should be determined in a way to guarantee the global minimum. In accordance with this purpose, transit time and resistivity readings corresponding to the estimated baseline can be taken as initial values. Matrix transit times are available for different rock types. Mineral composition of the rocks can be determined to figure out the distribution of the matrix transit times. Based on these observations, a proper initial value for matrix transit time can be specified. Passey et al. in their study proposes values for $a_{1}$ and $a_{3}$, and $a_{4}$ in Eq. (3) [11]. These values might be taken as initial values. Apart from these, specification of proper initial values is achieved by means of trial-and-error method.

\section{RESULTS AND DISCUSSION}

Fig. 2-3 show the measured TOC values, and the TOC values as a result of application of traditional formulation and modified version with respect to depth for different wells. The measured values shown in Fig. 2 and Fig. 3 were obtained from the study of Passey et al. and Wang et al., respectively [11] [16]. Here, Passey's formulation denotes the traditional approach involving the utilization of Eq. (1) or Eq. (14) depending on the representation for maturity. The parameters were optimized using the measured TOC values corresponding to Well-A1 and Well-A2 for the first formation while all the available measured TOC values are utilized for parameter optimization for the second formation due to considerable departure from the measured TOC values in some intervals (Well-C2 and Well-D2). The modified and traditional formulations involve utilization of LOM (Eq. (18)) and $\mathrm{T}_{\max }$ (Eq. (17)) values for the first and second formations, respectively.

In Fig. 2, the well that is highly categorized to possess reservoir potential is "Well-A1". For other wells the reservoir potentiality is varying among each other throughout the depth intervals, for example, for well "Well-C1", the lower interval is observed to have a high reservoir potentiality.

As it is observed in Fig. 3, for "Well-A2", the interval in between $1135-1155 \mathrm{~m}$ is considered to possess strong reservoir properties from the TOC potential standing point of view. The rest of the wells are noted to have varying TOC potential in reference to each other, and can be categorized to be marginally possessing reservoir potentiality.

Table 1 and Table 2 show the tried cementation exponents $(\mathrm{m})$ and the corresponding optimized parameter values and root mean square errors (RMSE), normalized root mean square errors (NRMSE) and Pearson correlation coefficient (r). Implementation of the nonlinear regression yields the $a_{2}$ values in the 5th column while $a_{2}$ values in the 6 th column are obtained solving the Eq. (21) with $t_{m}\left(a_{1}\right), t_{l}$ and $\mathrm{Rl}$ values in the 2 th, 3 th and 4 th columns, respectively. The $a_{2}$ values in the 5 th and 6 th columns are compared in order to check the accuracy of the $t_{l}$ and $R_{l}$ combination estimated based on the corresponding readings from well logs. Table 3 and Table 4 show the mean and standard deviation of resistivity and transit time values for each well.

Table 1. The tried cementation exponents $(\mathrm{m})$ and the corresponding optimized parameter values, root mean square errors (RMSE), normalized root mean square errors (NRMSE) and Pearson correlation coefficient (r) for the first formation.

\begin{tabular}{ccccccccccc}
\hline$m$ & $t_{m}\left(a_{1}\right)$ & $t_{l}$ & $R_{l}$ & $a_{2}$ & $a_{2}$ & $a_{3}$ & $a_{4}$ & $R M S E$ & NRMSE & $r$ \\
\hline 1.40 & 68.353 & 99 & 0.7 & 1.905 & 1.926 & 2.633 & -0.234 & 1.419 & 0.266 & 0.897 \\
1.80 & 60.172 & 99 & 0.7 & 2.693 & 2.706 & 2.478 & -0.212 & 1.283 & 0.241 & 0.912 \\
2.00 & 55.869 & 99 & 0.7 & 3.104 & 3.115 & 2.423 & -0.204 & 1.258 & 0.236 & 0.915 \\
2.40 & 46.988 & 99 & 0.7 & 3.955 & 3.964 & 2.340 & -0.193 & 1.244 & 0.233 & 0.916 \\
3.00 & 33.236 & 99 & 0.7 & 5.291 & 5.299 & 2.258 & -0.181 & 1.254 & 0.235 & 0.916 \\
4.00 & 9.701 & 99 & 0.7 & 7.641 & 7.648 & 2.179 & -0.170 & 1.285 & 0.241 & 0.914 \\
& & & Passey's: & 2.237 & -0.179 & 1.326 & 0.249 & 0.913 \\
\hline
\end{tabular}

Table 2. The tried cementation exponents $(\mathrm{m})$ and the corresponding optimized parameter values, root mean square errors (RMSE), normalized root mean square errors (NRMSE) and Pearson correlation coefficient (r) for the second formation.

\begin{tabular}{ccccccccccc}
\hline$m$ & $t_{m}\left(a_{1}\right)$ & $t_{l}$ & $R_{l}$ & $a_{2}$ & $a_{2}$ & $a_{3}$ & $a_{4}$ & $R M S E$ & $N R M S E$ & $r$ \\
\hline 1.40 & 49.553 & 61 & 10 & 2.482 & 2.482 & $43.348-16.217$ & 1.658 & 0.435 & 0.796 \\
1.80 & 47.265 & 61 & 10 & 3.055 & 3.048 & $41.582-15.557$ & 1.616 & 0.424 & 0.808 \\
2.00 & 46.036 & 61 & 10 & 3.355 & 3.350 & $40.902-15.305$ & 1.604 & 0.421 & 0.812 \\
2.40 & 43.471 & 61 & 10 & 3.982 & 3.985 & $39.841-14.911$ & 1.587 & 0.416 & 0.816 \\
3.00 & 39.456 & 61 & 10 & 4.981 & 5.000 & $38.746-14.506$ & 1.575 & 0.413 & 0.819 \\
4.00 & 32.497 & 61 & 10 & 6.773 & 6.820 & $37.637-14.098$ & 1.566 & 0.411 & 0.821 \\
& & & Passey's: & $34.670-13.012$ & 1.569 & 0.411 & 0.820 \\
\hline
\end{tabular}

Table 3. The mean and standard deviation of resistivity and transit time values for the first formation.

\begin{tabular}{cccccc}
\hline & Well-A1 & Well-B1 & Well-C1 & Well-D1 & Well-E1 \\
\hline Mean $(\Delta t)$ & 127.083 & 104.900 & 88.286 & 103.550 & 98.133 \\
Standard Deviation $(\Delta t)$ & 10.210 & 9.586 & 11.164 & 16.311 & 9.824 \\
Mean $(R)$ & 0.793 & 3.044 & 16.366 & 3.378 & 3.348 \\
Standard Deviation $(R)$ & 0.172 & 0.891 & 16.549 & 1.368 & 1.965 \\
\hline
\end{tabular}

Table 4. The mean and standard deviation of resistivity and transit time values for the second formation.

\begin{tabular}{cccccc}
\hline & Well-A2 & Well-B2 & Well-C2 & Well-D2 & Well-E2 \\
\hline Mean $(\Delta t)$ & 78.553 & 89.530 & 64.937 & 67.276 & 74.730 \\
Standard Deviation $(\Delta t)$ & 13.563 & 1.975 & 6.170 & 4.022 & 2.805 \\
Mean $(R)$ & 19.269 & 6.308 & 99.385 & 13.690 & 87.037 \\
Standard Deviation $(R)$ & 10.794 & 1.281 & 22.114 & 5.139 & 58.241 \\
\hline
\end{tabular}

As can be seen from the Table 1 and Table 2, altering the $m$ value does cause very little changes in the RMSE and NRMSE values. Actually, the main affected parameter 


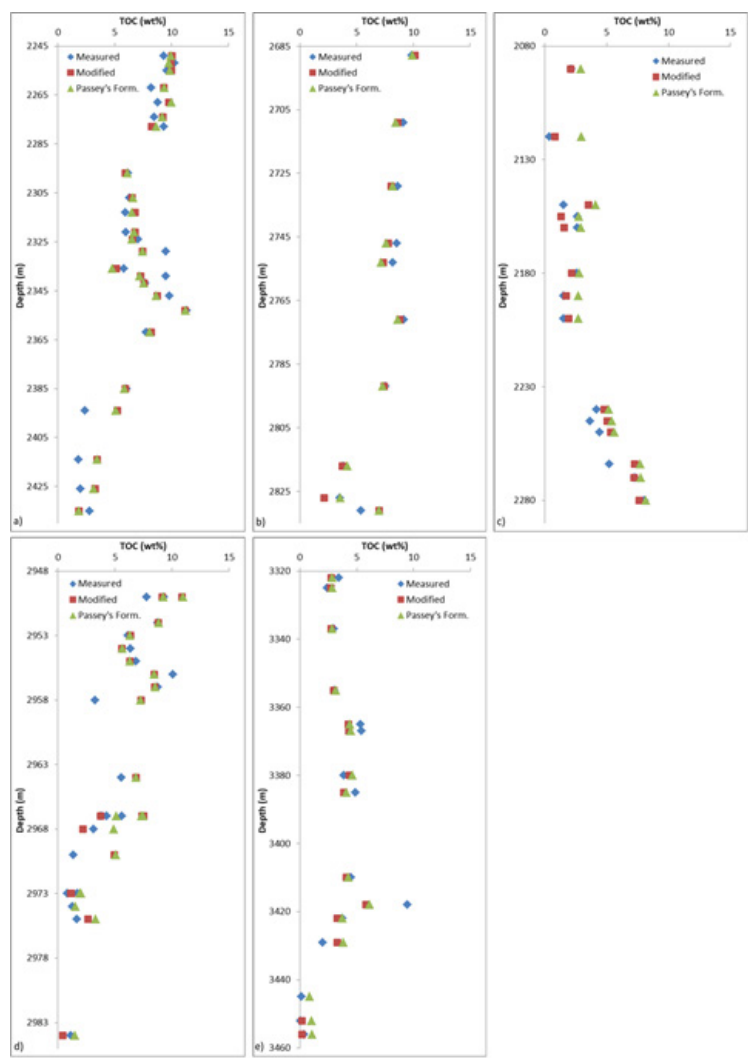

Figure 2. Comparison of the measured TOC values with the TOC values computed using modified version and traditional formulation for a) Well-A1, b) Well-B1, c) Well-C1, d) Well-D1, e) Well-E1.

is matrix transit time. Hence, an appropriate cementation exponent and matrix transit time combination is to be selected based on RMSE or NMRSE values and the previous observations. Cementation exponent of 2.4 and 2 and the corresponding optimized values were selected to calculate TOC in the first and second formations, respectively. The transit time values are close to the calculated transit time based on the mineral composition of shale in the study of Wang et al. [16], and the values of the determined cementation exponents are close to the generally observed cementation exponent values (around 2). On this respect, it can be asserted that the determined combinations satisfy the expectations. Note that the selected cementation exponent and transit time combination for the first formation yields the lowest RMSE and NRMSE values among the tried combinations.

Comparison of the $a_{2}$ values in the 5 th and 6 th columns reveals that the optimized $a_{2}$ values shown the 5 th column of the Table 1 and Table 2 can be attained with the single baseline transit time and resistivity combination which is also consistent to the combination to be obtained with the help of well logs.

In the study of Passey et al. [11], the logarithm of resistivity is plotted against sonic transit time using the so-

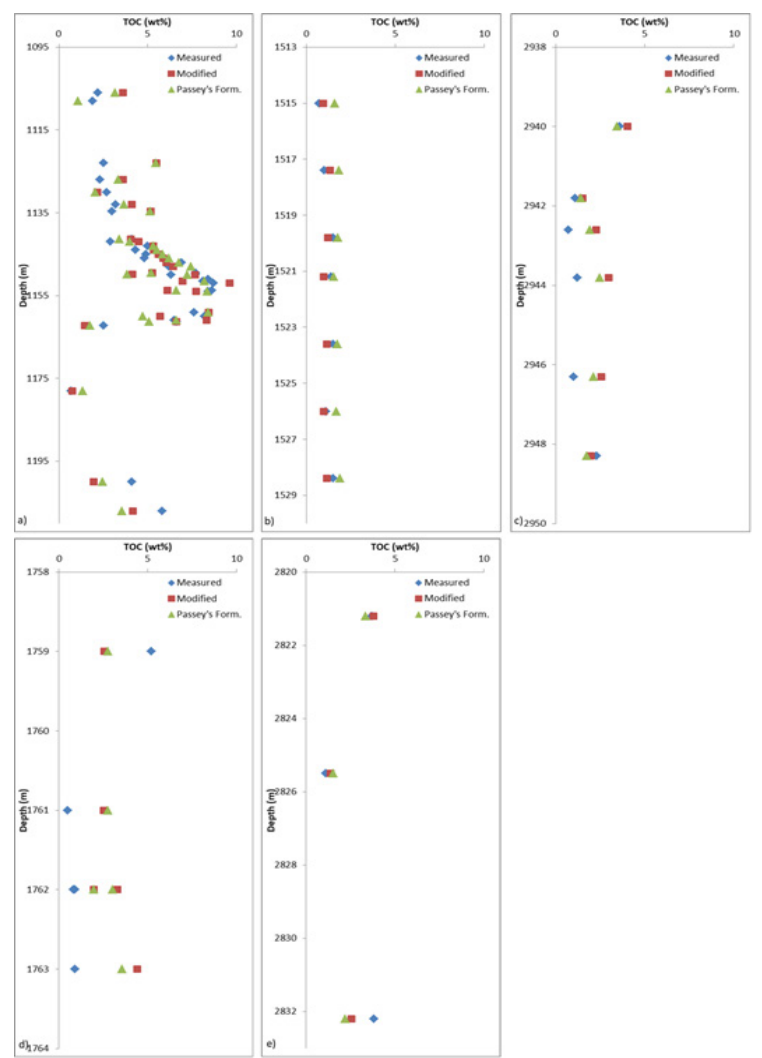

Figure 3. Comparison of the measured TOC values with the TOC values computed using modified version and traditional formulation for a) Well-A2, b) Well-B2, c) Well-C2, d) Well-D2, e) Well-E2.

nic porosity relationship proposed by Magara [20] and the boundaries are marked on the plot. It is observed the best line (drawn to fit the curve to form a linearship between the logarithm of resistivity and transit time as a requirement of Passey's method) deviates from the plot basically in the range of $70-90 \mu \mathrm{sec} / \mathrm{ft}$. In this study, the same types of plots are constructed for the two formations considered. In Fig. 4, the straight line corresponding to traditional formulation is coincided with the actual curve corresponding to modified version close baseline transit time and logarithmic resistivity. Note that actual curve is formed by means of Archie equation (Eq. (7)) and rectangular markers indicate the transit time values (training values) used in the optimization process. As it can be seen from Fig. 4, the best line deviates from the actual curve basically below $90 \mu \mathrm{sec} / \mathrm{ft}$ for the first formation. This range basically is compatible with the range for Well-C1 (Table 3). It is seen that, for well-C1, the modified version generally yields better match with the measured TOC values (Fig. 2) and results in smaller RMSE value in comparison to traditional formulation. RMSE is calculated as 1.034 $(\mathrm{r}=0.910)$ and $1.436(\mathrm{r}=0.914)$ by means of the application of the modified version and traditional formulation, respectively. For the second formation, the difference between the slopes of the curves is highly low between 60-70 $\mu \mathrm{sec} / \mathrm{ft}$ (Fig. 5). To increase the difference, only the values corresponding to the transit time values higher 
than $70 \mu \mathrm{sec} / \mathrm{ft}$ were utilized for optimization. The resultant plot is shown in Fig. 6. Note that the straight line deviates considerably from the actual curve between 60-70 $\mu \mathrm{sec} /$ $\mathrm{ft}$ different from the previous case. This range is basically compatible with the range for Well-C2 and Well-D2 (Table 4). The deviation actually reflects on the calculated TOC values for Well-C2 and Well-D2 in favor of the modified version as expected. In this case, RMSE is calculated as 1.790 $(r=0.218)$ and $2.306(r=-0.0186)$ by means of the modified version and traditional formulation, respectively. Note that previous values are $1.879(\mathrm{r}=0.133)$ and $1.616(\mathrm{r}=0.260)$ for the modified version and traditional formulation, respectively. Furthermore, it should be noted that the estimation of TOC contents for the intervals corresponding to the transit times above $70 \mu \mathrm{sec} / \mathrm{ft}$ were not considered for improvement although the straight line considerably deviates from the actual curve above $70 \mu \mathrm{sec} / \mathrm{ft}$. This is related to the result of the optimization method, which reveals that relationship between logarithmic resistivity and transit time is not significant in that interval. These comparisons illustrate the significance of utilization of the modified version to achieve better results for the intervals where the validity of the assumption about linearity is not satisfactory.

Passey et al. in their study emphasize the effect of clay minerals on the resistivity of the formations apart from the effect of porosity [11]. They apply Waxman-Smith equation to realize the effect of clay minerals on the relationship between logarithm of resistivity and sonic transit time. It is proved that the clay minerals lowers the resistivity of the rocks however the curves plotted for different clay concentrations are shown to be almost parallel to each other, that is to say these curves have almost same slopes. Beside this, the effect of igneous rocks and salty zones on the resistivity log should be considered [11]. Invariance of the curve slopes in consideration of change in mineral composition being valid, the modified version requires basically the accurate determination of the resistivity and transit time values corresponding to the baseline. Considerable deviations from the measured TOC values are especially observed for Well-C2 and Well-D2 for the second formation. Whether these deviations stem from the measurement errors is indefinite.

The comparison with the measured TOC and the calculated RMSE values prove the accuracy of the modified versions. It is advisable to utilize the traditional formulation with the modified version to detect the deviations of the linear curve from the actual curve, which stem from the assumption of linear relationship between logarithm of resistivity and transit time in the traditional approach. The results indicate that better estimation of TOC can be made utilizing the modified version for these sections lack of training. The magnitude of resistivity and transit time corresponding to the baseline vary depending on the mineral composition

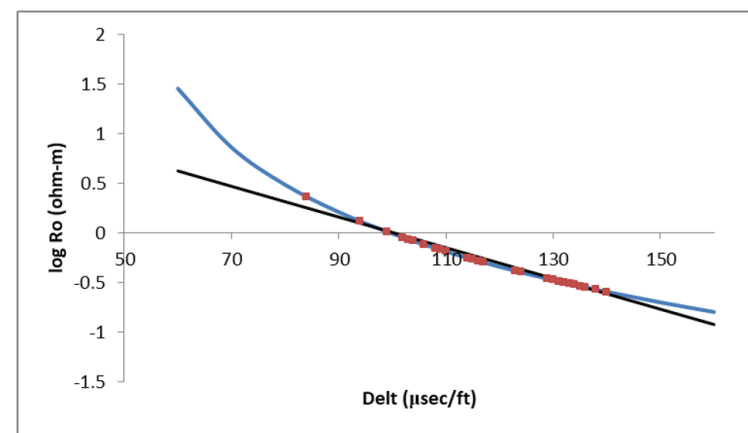

Figure 4. The comparison of the actual curve based on the Archie equation and the straight line corresponding to traditional formulation for the first formation.

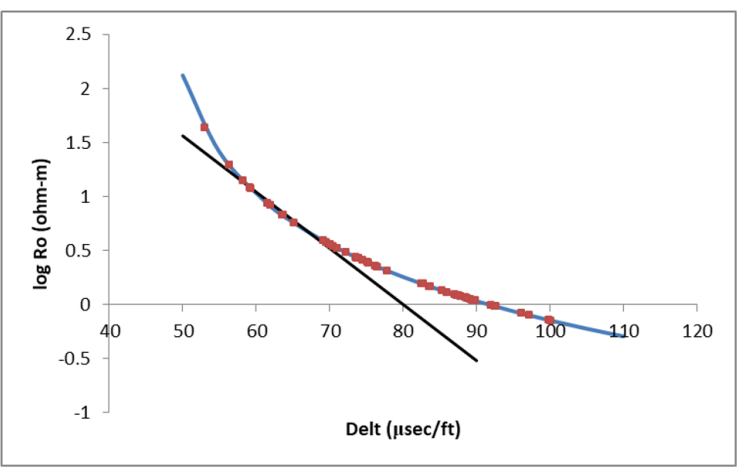

Figure 5. The comparison of the actual curve based on the Archie equation and the straight line corresponding to traditional formulation for the second formation as a result of first trial.

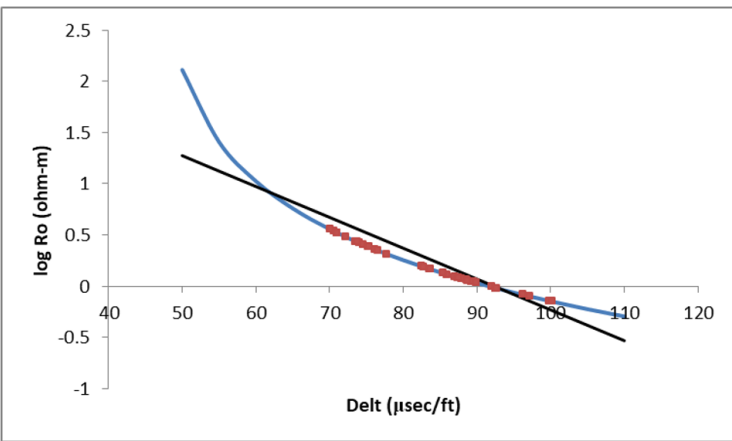

Figure 6. The comparison of the actual curve based on the Archie equation and the straight line corresponding to traditional formulation for the second formation as a result of second trial.

of the matrix. To enhance the solution in this case, the intervals should be partitioned and matched based on the baseline resistivity and transit time values and regression should be applied for each partitioned interval group. By this way, each interval group will correspond to specific optimized parameters. Sufficient data should be provided to perform this methodology properly.

The change in porosity is another factor to be accounted for. The effect of increase in porosity is similar to the effect of increase in TOC in mature source rock [11]. Hence, it may be hard to distinguish these two effects. Utilizing 
the measured porosity values can be helpful in this case. On the contrary, the effect of decrease in porosity can be easily distinguishable with increase in resistivity and reduction in transit time.

\section{CONCLUSION}

This study involves the modification of the traditional approach to enhance TOC estimation. The modified formulations are observed to be appropriate for the nonlinear regression application. The comparison with the measured TOC and the calculated RMSE values prove the accuracy of the modified versions. The comparisons illustrate the significance of utilization of the modified version to achieve better results for the intervals where the validity of the assumption of linear relationship between logarithmic resistivity and sonic transit time is not satisfactory. It is advisable to utilize the traditional formulation with the modified version to detect the deviations which stem from this assumption. With the methodology presented in this research work the TOC of unconventional reservoirs and source rocks can be more accurately calculated.

\section{ACKNOWLEDGEMENTS}

In this study, the related tool of Matlab software was utilized for nonlinear regression application. Authors are thankful to the corresponding author's faculty for providing the academic version of the Matlab software.

\section{NOMENCLATURE}

$\Delta_{t}=$ sonic transit time on the logs, $\mu \mathrm{sec} / \mathrm{ft}$

$\Delta_{t f}=$ sonic transit time of fluid, $\mu \mathrm{sec} / \mathrm{ft}$

$\Delta_{t l}=$ sonic transit time on the baseline, $\mu \mathrm{sec} / \mathrm{ft}$

$\Delta_{t m}=$ sonic transit time of matrix, $\mu \mathrm{sec} / \mathrm{ft}$

$a=$ parameter to be optimized

$a_{1}=$ parameter to be optimized

$a_{2}=$ parameter to be optimized

$a_{3}=$ parameter to be optimized

$a_{4}=$ parameter to be optimized

$b=$ parameter to be optimized

$c=$ parameter to be optimized

$d=$ parameter to be optimized

$G R_{l}=$ base line gamma ray log, API Unit

$G R$ = gamma ram log, API Unit

$J=$ jacobian matrix

$J^{T}=$ transpose of jacobian matric

$L O M=$ level of organic maturity

$m=$ cementation exponent of the rock

$p=$ slope

$r=$ Pearson correlation coefficient

$R=$ resistivity log, ohm-m
$R_{l}=$ resistivity reading on the baseline, ohm-m

$R_{o}=$ resistivity of the brine saturated zone, ohm-m

$R_{w}=$ resistivity of the brine, ohm-m

$T_{\text {max }}=$ maximum pyrolysis temperature, ${ }^{\circ} \mathrm{C}$

TOC $=$ total organic carbon content, wt $\%$

$x=$ input function data

$y=$ measured parameter

Greek:

$\lambda=$ damping factor

$\phi=$ porosity, fraction

$\delta=$ vector to update $\beta$

$\beta=$ parameter to be optimized

\section{CONFLICT OF INTEREST}

Authors approve that to the best of their knowledge, there is not any conflict of interest or common interest with an institution/organization or a person that may affect the review process of the paper.

\section{AUTHOR CONTRIBUTION}

Can Polat and Tuna Eren: Conceptualization, Methodology, Software. Can Polat: Data Curation, Writing- Original Draft Preparation. Can Polat and Tuna Eren: Visualization, Investigation. Can Polat: Supervision. Can Polat and Tuna Eren: Writing- Reviewing and Editing.

\section{References}

1. Ma YZ, Holditch SA. Unconventional Oil and Gas Resources Handbook: Evaluation and Development. Gulf Professional Publishing, USA, 2015.

2. Tuft AL. Unconventional Oil and Shale Gas: Growth, Extraction, and Water Management Issues (Energy Science, Engineering and Technology). Nova Science Pub Inc, UK, 2015.

3. Mahmood MF, Ahmad Z, Ehsan M. Total Organic Carbon Content and Total Porosity Estimation in Unconventional Resource Play Using Integrated Approach Through Seismic Inversion and Well Logs Analysis within the Talhar Shale, Pakistan. Journal of Natural Gas Science and Engineering 52 (2018) 13-24.

4. Edward D, Holstein E. Petroleum Engineering Handbook Volume V: Reservoir Engineering and Petrophysics. Society of Petroleum Engineers, Richardson, Texas, 2007.

5. Zou C. Unconventional Petroleum Geology, second ed. Elsevier Inc, USA, 2017.

6. Ahmed U, Meehan DN. Unconventional Oil and Gas Resources: Exploitation and Development Gulf Professional Publishing, USA, 2016.

7. Steiner S, Ahsan SA, Raina I, Dasgupta S, Lis GP. Interpreting Total Organic Carbon TOC in Source Rock Oil Plays. Paper presented at the Abu Dhabi International Petroleum 
Exhibition \& Conference, Abu Dhabi, UAE, 7-10 November, SPE, pp. 1-18, 2016.

8. Thomas BM. Geochemical Analysis of Hydrocarbon Occurrences in Northern Perth Basin, Australia. American Association of Petroleum Geologists Bulletin 63 (1979) 1092-1107.

9. Mahmoud A, Elkatatny S, Mahmoud M, Abouelresh M, Abdulraheem A, Ali A. Determination of the Total Organic Carbon (TOC) Based on Conventional Well Logs Using Artificial Neural Network. International Journal Coal Geology 179 (2017) 72-80.

10. Huang $R$, Wang $Y$, Cheng S, Liu S, Cheng L. Selection of Logging-Based TOC Calculation Methods for Shale Reservoirs: A Case Study of the Jiaoshiba Shale Gas Field in the Sichuan Basin. Natural Gas Industry B 2 (2015) 155-161.

11. Passey QR, Creaney S, Kulla JB, Moretti FJ, Stroud JD. A Practical Model for Organic Richness from Porosity and Resistivity Logs. American Association of Petroleum Geologists Bulletin 74 (1990) 1777-1794.

12. Decker AD, Hill DG, Wicks DE. Log-based Gas Content and Resource Estimates for the Antrim Shale, Michigan Basin. Paper presented at the Low Permeability Reservoirs Symposium, Denver, Colorado, 26-28 April, SPE, pp. 659669, 1993.

13. Levenberg K. A Method for the Solution of Certain Problems in Least Squares. Quarterly of Applied Mathematics 2
(1944) 164-168.

14. Marquardt D. An Algorithm for Least-Squares Estimation of Nonlinear Parameters. SIAM Journal on Applied Mathematics 11 (1963) 431-441.

15. Archie GE. The Electrical Resistivity Log as an Aid in Determining Some Reservoir Characteristics. Transactions of the AIME, 146 (1942) 54-62.

16. Wang P, Chen Z, Pang X, Hu K, Sun M, Chen X. Revised Models for Determining TOC in Shale Play: Example from Devonian Duvernay Shale, Western Canada Sedimentary Basin. Marine and Petroleum Geology 70 (2016) 304-319.

17. Lourakis MIA. A Brief Description of the LevenbergMarquardt Algorithm Implemented by levmar. https:// www.researchgate.net/publication/239328019_A_Brief_ Description_of_the_Levenberg-Marquardt_Algorithm_ Implemened_by_levmar. Accessed June 3, 2021.

18. Wikipedia contributors. Levenberg-Marquardt Algorithm. Wikipedia, The Free Encyclopedia. https://en.wikipedia. org/wiki/Levenberg\%E2\%80\%93Marquardt_algorithm. Accessed February 12, 2021.

19. Fletcher R. A modified Marquardt Subroutine for Nonlinear Least Squares. Atomic Energy Research Establishment, Rpt. AERE-R 6799, Harwell, 1971.

20. Magara K. Compaction and Fluid Migration: Practical Petroleum Geology Developments in Petroleum Science, Vol. 9, Elsevier Science\&Technology, Amsterdam, 1978. 